\title{
540.
}

\section{ON A PROPERTY OF THE TORSE CIRCUMSCRIBED ABOUT TWO QUADRIC SURFACES.}

[From the Messenger of Mathematics, vol. I. (1872), pp. 111, 112.]

THE property mentioned by Mr Townsend in his paper( $\left.{ }^{1}\right)$ in the August No., "On a Property in the Theory of Confocal Quadrics," may be demonstrated in a form which, it appears to me, better exhibits the foundation and significance of the theorem.

Starting with two given quadric surfaces, the torse circumscribed about these touches each of a singly infinite series of quadric surfaces, any two of which may be used (instead of the two given surfaces) to determine the torse; in the series are included four conics, one of them in each of the planes of the self-conjugate tetrahedron of the two given surfaces; and if we attend to only two of these conics, the two conics are in fact any two conics whatever, and the torse is the circumscribed torse of the two conics; or, what is the same thing, it is the envelope of the common tangent-planes of the two conics.

Consider now two conics $U, U^{\prime}$, the planes of which intersect in a line $I$; and let $I$ meet $U$ in the points $L, M$, and meet $U^{\prime}$ in the points $L^{\prime}, M^{\prime}:$ take $A$ the pole of $I$ in regard to the conic $U$, and $A^{\prime}$ the pole of $I^{\prime}$ in regard to the conic $U^{\prime}$.

Take $T$ any point on $I$, and draw $T P$ touching $U$ in $P$, and $T P^{\prime}$ touching $U^{\prime}$ in $P^{\prime}$ : the points $P, P^{\prime}$ may be considered as corresponding points on the two conics respectively.

Join $A P$ and produce it to meet the line $I$ in $G$; the line $A P G$ is in fact the polar of $T$ in regard to the conic $U$ (for $T$ being a point on $I$, the polar of $T$ passes through $A$; and this polar also passes through $P$ ); that is, the points $T, G$ and $L, M$ are harmonics on the line $I$; whence also, in the plane of the conic $U^{\prime}$,

[1 Messenger of Mathematics, same volume, pp. 49, 50.] 
the lines $P^{\prime} G, P^{\prime} T$ and $P^{\prime} L, P^{\prime} M$ are harmonic lines through the point $P^{\prime}$. It thus appears that in the particular case where the points $L, M$ are the foci of the conic $U^{\prime}$, the line $P^{\prime} G$ is the normal at the point $P^{\prime}$; and we may say in general that $P^{\prime} G$ is the quasi-normal at the point $P^{\prime}$ of the conic $U^{\prime}$.

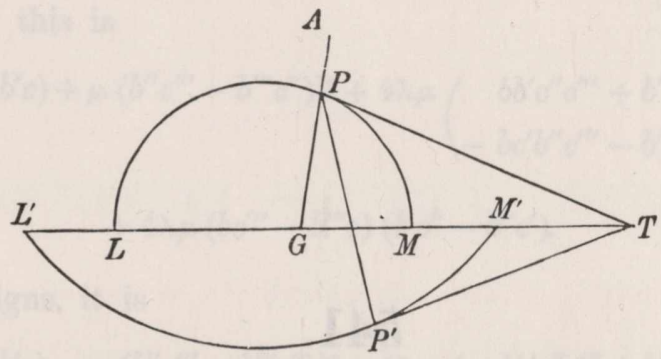

Consider now the torse circumscribed about the conics $U, U^{\prime}$; the plane $P T P^{\prime}$ will represent any plane, and the line $P P^{\prime}$ any line of this torse: projecting on the plane of $U^{\prime}$ with the point $A$ as centre of projection, the projection of $P P^{\prime}$ is the line $P^{\prime} G$; which, as just seen, is the quasi-normal of the conic $U^{\prime}$ at the point $P^{\prime}$.

The projection of the cuspidal curve is the envelope of line $P^{\prime} G$, which is the projection of the generating line $P P^{\prime}$ of the torse-viz. this envelope is the quasievolute of the conic $U^{\prime}$; which is the theorem in question. 